\title{
Applying recursive numerical integration techniques for solving high dimensional integrals
}

\section{Andreas Ammon ${ }^{e}$, Alan Genz ${ }^{b}$, Tobias Hartung $^{c}$, Karl Jansen $^{a}$, Hernan Leövey ${ }^{d}$, Julia Volmer ${ }^{\star a}$}

${ }^{a} N I C, D E S Y$

Platanenallee 6, D-15738 Zeuthen, Germany

${ }^{b}$ Department of Mathematics, Washington State University

Pullman, WA 99164-3113 USA

${ }^{c}$ Department of Mathematics, King's College London

Strand, London WC2R 2LS, United Kingdom

${ }^{d}$ Institut für Mathematik, Humboldt-Universität zu Berlin

Unter den Linden 6, D-10099 Berlin

${ }^{d}$ IVU Traffic Technologies AG

Bundesallee 88, 12161 Berlin, Germany

Email: andreas.ammon@desy.de, genz@math.wsu.edu, tobias.hartung@kcl.ac.uk, karl.jansen@desy.de, leovey@math.hu-berlin.de, julia.volmer@desy.de

\begin{abstract}
The error scaling for Markov-Chain Monte Carlo techniques (MCMC) with $N$ samples behaves like $1 / \sqrt{N}$. This scaling makes it often very time intensive to reduce the error of computed observables, in particular for applications in lattice QCD. It is therefore highly desirable to have alternative methods at hand which show an improved error scaling. One candidate for such an alternative integration technique is the method of recursive numerical integration (RNI). The basic idea of this method is to use an efficient low-dimensional quadrature rule (usually of Gaussian type) and apply it iteratively to integrate over high-dimensional observables and Boltzmann weights. We present the application of such an algorithm to the topological rotor and the anharmonic oscillator and compare the error scaling to MCMC results. In particular, we demonstrate that the RNI technique shows an error scaling in the number of integration points $m$ that is at least exponential.
\end{abstract}

34th annual International Symposium on Lattice Field Theory

24-30 July 2016

University of Southampton, UK

${ }^{*}$ Speaker. 


\section{Introduction}

For evaluation of the high dimensional path integrals in numerical simulations of statistical physics and lattice-QCD [1] mainly Markov-Chain Monte Carlo methods (MCMC) are used.

If we have a model with discrete variables $\left\{x_{1}, \ldots, x_{d}\right\}, x_{i} \in D \subset \mathbb{R}$ and an action $S[x] \equiv$ $S\left(x_{1}, \ldots, x_{d}\right)$, we can measure the expectation value of an observable $O[x]$ in this model by

$$
\langle O\rangle=\frac{\int_{D^{d}} \mathrm{~d} x O[x] \mathrm{e}^{-S[x]}}{\int_{D^{d}} \mathrm{~d} x \mathrm{e}^{-S[x]}} .
$$

These integrals are usually impossible to approximate with reasonable sample sizes by means of direct sampling because $d$ is large. MCMC methods can be applied to large dimensions $d$ because they often choose $N$ sampling configurations $[x]$ from a probability distribution dependent on $\mathrm{e}^{-S[x]}$. On the other side the error of $\langle O\rangle$ scales weakly, namely like $\frac{1}{\sqrt{N}}$. Additionally, the consecutively chosen sampling points have a dependence and these autocorrelations can lead to large errors since they necessitate very long MCMC runs. Therefore it is highly desirable to look for alternative methods to improve the weak error scaling, especially for very time-intensive computations as they are done for example in lattice-QCD.

One alternative approach, the Recursive Numerical Integration (RNI) method [2, 3], promises to overcome both MCMC drawbacks, at least in principle. RNI is a polynomially exact method, not of statistical nature, therefore we directly avoid autocorrelations and the theoretical predictions for the error scaling are usually at least exponential. In this method we compute numerator and denominator in (1.1) separately. Similar to MCMC we also exploit the weighting function $\mathrm{e}^{-S[x]}$ but not as a probability density but by using its structure to simplify the integrals and finally apply Gaussian quadrature to solve them numerically to get a polynominally exact solution. The simplification of course depends a lot on the action itself and therefore is model dependent. Here we apply the method to the topological oscillator to obtain a first test whether the predicted improved error scaling can indeed be reached.

\section{Recursive Numerical Integration}

We use the principle of Recursive Numerical Integration (RNI) $[2,3]$ to derive the final formula we use to compute each of the two main integrals in (1.1). The derivation consists of three main steps. First we identify a structure in the integrand, secondly we integrate recursively, and finally choose a quadrature rule to perform each integration numerically. The last paragraph in this section is dedicated to the discussion of the differences in computing the two integrals in (1.1)

Integrand Structure Throughout the whole paper we look at physical 1-dimensional models, schematically shown at the top of figure 1 , with $d$ lattice points, only next-neighbor couplings $f_{i}$ (which are in general different from each other) and periodic boundaries. Because in addition all considered observables are of algebraic nature we can write each of the two integrals in (1.1) in the form

$$
I=\int_{D} \mathrm{~d} x_{1} \ldots \int_{D} \mathrm{~d} x_{d} \prod_{i=1}^{d} f_{i}\left(x_{i}, x_{i+1}\right)
$$




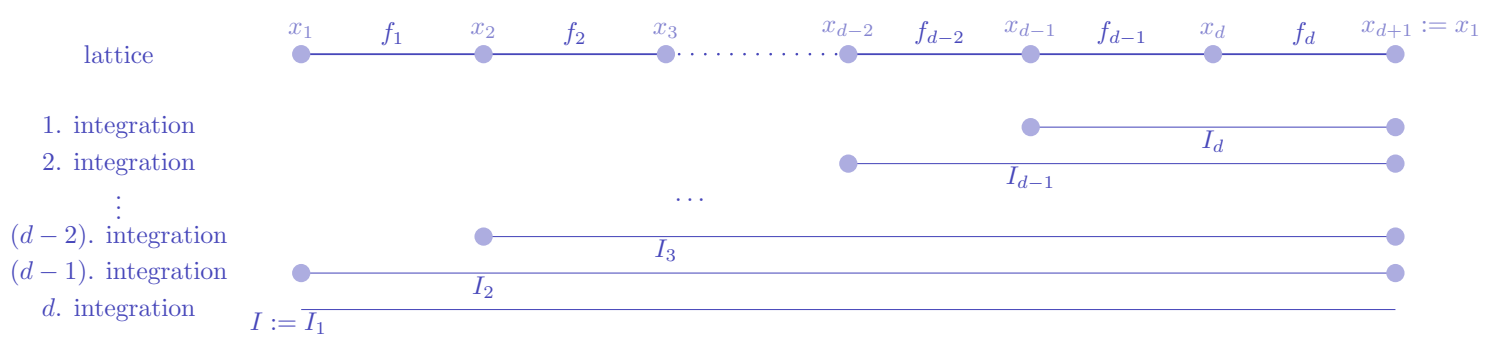

Figure 1: Visualization of the recursive integration steps for a 1-dimensional lattice with $d$ points $x_{i}$, nextneighbor couplings $f_{i}$ and periodic boundaries: How to consecutively calculate $I_{i}, i \in\{d, d-1, \ldots, 1\}$ by integrating out the lattice variable $x_{i}$ (shown in the first row) and finally arrive at the desired integral $I$.

Recursive Integration We can reorder expression (2.1). Because of the next-neighbor coupling every lattice point $x_{i}$ appears only twice, in $f_{i}$ and $f_{i-1}$, and $I$ can be written

$$
I=\underbrace{}_{D} \mathrm{~d} x_{1} \ldots \underbrace{(\int_{D} \mathrm{~d} x_{d-2} f_{d-3}\left(x_{d-3}, x_{d-2}\right) \cdot \underbrace{\left.(\int_{D} \mathrm{~d} x_{d-1} f_{d-2}\left(x_{d-2}, x_{d-1}\right) \cdot \underbrace{\left(\int_{D} \mathrm{~d} x_{d} f_{d-1}\left(x_{d-1}, x_{d}\right) \cdot f_{d}\left(x_{d}, x_{d+1}\right)\right)}_{I_{d}})\right)}_{I_{d-1}}}_{I_{d-2}} .
$$

This recursive integration process is visualized in the lower part of figure 1. The first integration we can perform is the integral standing at the right end of equation (2.2) over $f_{d-1}$ and $f_{d}$. Here we integrate out $x_{d}$ and call the resulting integral $I_{d}$. $I_{d}$ has two open indices, $x_{d-1}$ and $x_{d+1}$. It is visualized in the figure as an uninterrupted line including $f_{d-1}$ and $f_{d}$ from knot $x_{d-1}$ to $x_{d+1}$.

In the second integration step we integrate out $x_{d-1}$. Here we integrate over the already calculated $I_{d}$ and the new $f_{d-2}$, which, as $I_{d}$, depends on $x_{d-1}$. This procedure is repeated until we have integrated out all $x_{i}$, where for the last integration over $x_{1}$ we just have to integrate over $I_{2}\left(x_{1}, x_{1}\right)$.

Numerical Integration For numerical integration we want to approximate each of the above integrals with some integrand $g(x)$ by a weighted sum (quadrature) $\int_{D} \mathrm{~d} x g(x) \approx \sum_{r=1}^{m} w^{r} g\left(x^{r}\right)$, where we evaluate the integrand at specific mesh points $x^{r}$ and weight this result by corresponding weights $w^{r}{ }^{1}$ Applying this approximation to each integral in (2.2) leads to a transformation of $\int_{D} \mathrm{~d} x_{i} \rightsquigarrow \sum_{r=1}^{m} w_{i}^{r}$ and $f_{i}\left(x_{i}, x_{i+1}\right) \rightsquigarrow M_{i}\left(x_{i}^{j}, x_{i+1}^{k}\right)=: M_{i}^{j, k}$. We apply this approximation to the first integration step to get

$$
I_{d}\left(x_{d-1}^{s}, x_{1}^{t}\right) \approx \sum_{r=1}^{m} w^{r} M_{d-1}^{s, r} M_{d}^{r, t}=(\underbrace{M_{d-1} \cdot \operatorname{diag}\left(w^{1}, w^{2}, \ldots, w^{m}\right)}_{\widetilde{M}_{d-1}} \cdot M_{d})^{s, t},
$$

which is just the $(s, t)$ entry of the matrixproduct in brackets. This is valid for all integration mesh points of $x_{d-1}^{s}$ and $x_{1}^{t}, s, t \in\{1, \ldots, m\}$.

\footnotetext{
${ }^{1}$ The superscript here is used to distinguish these integration indices from the discretization indices and should not be interpreted as some exponential.
} 
For each of the following integration steps $i \in\{2, \ldots, d-1\}$, we get an additional sum, weight and a matrix, which gives an additional $\widetilde{M}_{d-i+1}$ in the matrix product. Therefore after repeating this step $d-2$ times we arrive at $I_{2}\left(x_{1}^{t}, x_{1}^{t}\right) \approx\left(\left(\prod_{i=1}^{d-1} \widetilde{M}_{i}\right) M_{d}\right)^{t, t}$. Integrating finally over $x_{1}$ gives

$$
I=I_{1}=\int_{D} \mathrm{~d} x_{1} I_{2}\left(x_{1}, x_{1}\right) \approx \sum_{t=1}^{m} w^{t} I_{2}^{t, t}=\operatorname{tr}\left[\operatorname{diag}\left(w^{1}, \ldots, w^{m}\right) I_{2}^{t, t}\right]=\operatorname{tr}\left[\left(\prod_{i=1}^{d} \widetilde{M}_{i}\right)\right] .
$$

Because RNI is a deterministic method and we are mostly interested in the scaling of the error and not the value of the integration itself, we estimate the error of the integral $I(m)$ at some number of mesh points $m$ by a truncation error, i.e. by computing $I$ at a larger $m_{0}>m$ value and compute the difference $\Delta I(m)=\left|I(m)-I\left(m_{0}\right)\right|$.

At this point, we have not yet specified which quadrature to use. For every 1-dimensional integration $2 m$ parameters, the mesh points and weights, have to be determined. By using Gauss quadrature we approximate the integrand by a polynomial of degree $2 m-1^{2}$ and therefore obtain a polynomially exact solution to our integral. One valid choice for these polynomials are the Legendre polynoms of degree $m$. This is a good choice because the functions we look at are mostly exponentials which are approximated well by Legendre polynomials.

If the integrand $g(x)$ is not itself a polynomial of degree $2 m-1$ but sufficiently smooth in the integration range $D, g(x) \in C^{2 m}(D)$, the error scaling of the integral approximation by using Gauss quadrature is asymptotically (for large enough $m$ )

$$
\mathscr{O}\left(\frac{1}{(2 m) !}\right) \sim \mathscr{O}\left(\frac{\mathrm{e}^{2 m}}{\sqrt{2 \pi 2 m}(2 m)^{2 m}}\right)=\mathscr{O}\left(\exp [-2 m \ln m] \cdot \frac{1}{\sqrt{m}}\right)
$$

where we used the Stirling formula to estimate the factorial.

Types of integrals There are two types of integrals we want to compute, the numerator and the denominator of equation (1.1). The denominator integrates the Boltzmann weight. Assuming isotropy of a given model we obtain here $f_{1}=f_{2}=\ldots=f_{d}$. Therefore also $M_{1}=M_{2}=\ldots=M_{d}$ and the final integral (2.4) simplifies to $I \approx \operatorname{tr}\left[(\widetilde{M})^{d}\right]$. This can be evaluated by either computing the eigenvalues of $\widetilde{M}$, raise them to the $d$ th power and sum them up or computing the $d$ th power of the matrix explicitly and take the trace. The choice of the approximating polynomial and the chosen quadrature rule are very important since $\widetilde{M}$ is a $m \times m$ matrix. Hence, the smaller $m$ is, the easier it is to compute $I$.

The numerator depends strongly on the observable. This is normally some summation and/or multiplication of the variables $x_{1}, \ldots, x_{d}$. In general this means we obtain different $f_{i}$ 's though most of them will still coincide in practice. Thus, we need to compute the trace of a product of at most $d$ matrices, some of which may be raised to a power less than $d$.

\section{Topological Oscillator}

In this section, we apply the discussed method of RNI to the topological oscillator. This simple physical 1-dimensional model shows some characteristic features of non-linear $\sigma$-models

\footnotetext{
${ }^{2}$ which has $2 m$ free coefficients
} 
and gauge theories, see e.g. $[4,5,6]$. It describes a particle with mass $M$ moving on a circle with radius $R$. The basic degree of freedom is an angle $\phi$ dependent on time $t$. The action of this system is the integration of the kinetic energy of the particle over a time period $T$. We discretize one time period in $d$ slices with spacing $a$. On each timeslice lives an angle $\phi_{i}=\phi\left(t_{i}\right), i \in\{1, \ldots, d\}$.

$$
S_{\text {continuum }}(\phi)=\frac{M R^{2}}{2} \int_{0}^{T} \mathrm{~d} t\left(\frac{\partial \phi}{\partial t}\right)^{2} \stackrel{\text { lattice }}{\Longrightarrow} S[\phi]=\frac{M R^{2}}{a} \sum_{i=1}^{d}\left(1-\cos \left(\phi_{i+1}-\phi_{i}\right)\right) .
$$

The right side of the above equation is one possibility for the discretized action. One characteristic quantity of this system is the topological charge. This is the number of complete revolutions of the rotor in the time period $T$,

$$
Q_{\text {continuum }}(\phi)=\frac{1}{2 \pi} \int_{0}^{T} \mathrm{~d} t\left(\frac{\partial \phi}{\partial t}\right) \quad \stackrel{\text { lattice }}{\Longrightarrow} Q[\phi]=\frac{1}{2 \pi} \sum_{i=1}^{d}\left(\phi_{i+1}-\phi_{i}\right) \bmod [-\pi, \pi) .
$$

A more physical observable to characterize a system is the width of the distribution $Q$ normalized by the time period $T$, the topological susceptibility $\chi$, with the continuum limit

$$
\chi[\phi]=\frac{\left\langle Q^{2}[\phi]\right\rangle}{T} \underset{a \cdot d=\mathrm{const}}{\stackrel{a \rightarrow 0, d \rightarrow \infty}{\longrightarrow}} \frac{1}{4 \pi^{2} M R^{2}} .
$$

\section{Numerical Results}

As a benchmark quantity we compute the topological susceptibility with the RNI method. We first test the correctness of the method by checking that we obtain the right correct continuum limit value. Then we look at the error scaling as a function of the number of integration steps we use. And finally we compare the error scaling and the cost of the computation with the optimal MCMC Cluster algorithm results by looking at the runtime of both methods on a standalone computer.

To demonstrate the correctness of the results computed with RNI we first fix the number of integration points to $m=120$ and $M R^{2}=0.25$ and computete the topological susceptability $\chi$ for different lattice spacings $a$. The expected continuum limit value, considering (3.3), is $\chi_{\text {continuum }}=$ $\frac{1}{\pi^{2}}$. The result of the computed $\chi$ subtracted by this expected theoretical value is shown in figure 2 and, as expected, it converges to zero at $a=0$, compare also [7].

Next we examine the error scaling of the RNI method for which we use the truncation error as discussed above. We choose $M R^{2}=0.25, a=0.4$ and a $\chi$ gauge value at $m_{0}=560$ to compute the truncation error for $\chi$ values at $m<500$. Results are shown in figure 3. We fit an exponential $\exp (-\mathrm{cm})$ to the data points in the range $220 \leq m \leq 480$, which appears as a straight line in figure 3 , where a logarithmic scale is used. The good agreement between the data and the exponential suggests that asymptotically the error scales at least exponentially fast. This is comparable with the expected error scaling for Gauss quadrature in (2.5). The expected weakening of the exponential decay by the additional $\ln m$ in the exponent and the $1 / \sqrt{m}$ behavior cannot be resolved here, probably because we are not yet fully in the asymptotic regime where (2.5) holds.

With an exponential error scaling RNI will clearly outperform any other method with an algebraic error behavior, especially the $1 / \sqrt{N}$ behavior of MCMC. An interesting question is whether for smaller, more practical values of $m$ RNI still gives better results than MCMC. Therefore we 


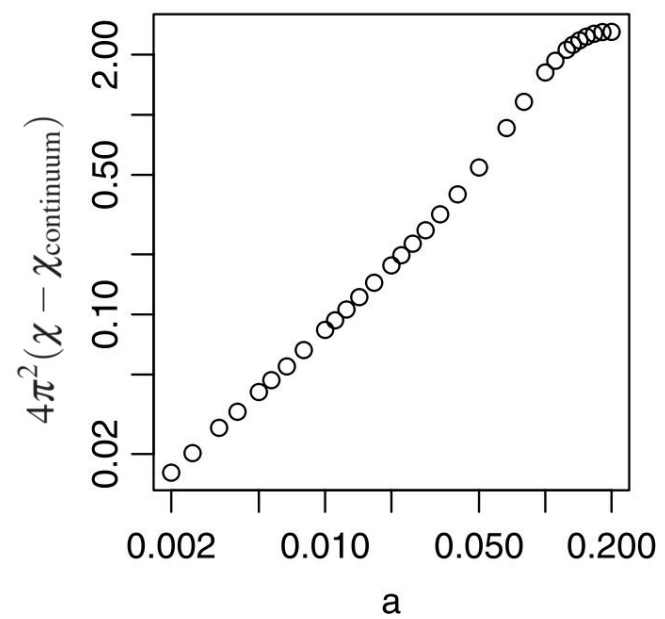

Figure 2: Continuum extrapolation of the topological susceptibility $\chi$ of the topological oscillator, computed with RNI.

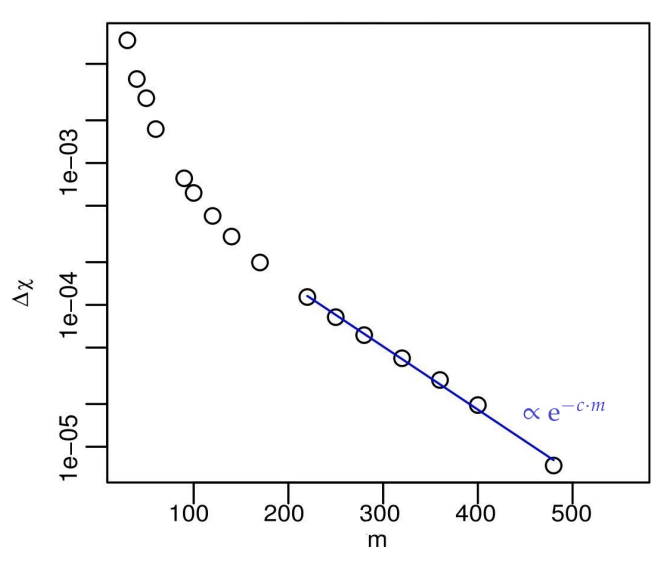

Figure 3: Error scaling of the topological susceptibility $\Delta \chi$ computed with RNI with number of integration points $m$. The blue line is an exponential fit in the range $220 \leq m \leq 480$.

compare MCMC and RNI directly. In the special case of the Topological Oscillator we can apply a specific kind of MCMC algorithm, the cluster algorithm [8]. This algorithm leaves the autocorrelation time almost constant when going to smaller lattice spacings and hence is an optimal algorithm for our system. Because both methods use different error scaling variables (number of mesh points $m$ for RNI and number of MCMC samples $N$ for the Cluster algorithm) we compare them by runtime $t$ on a standalone computer. For these computations we use $a=0.1$ and $M R^{2}=0.25$ for both methods and vary $N=10^{2} \ldots 10^{6}$ for MCMC and $m=10 \ldots 300$ for RNI. The runtime varies depending on other processes running on the computer, therefore we repeat every measurement 10 times to get an error estimation of the runtime. For the cluster algorithm, in addition, the size and distributions of the generated clusters can vary, leading to different runtimes of the algorithm. The error on the topological susceptibility can be estimated for the cluster algorithm by the slightly different topological susceptibility results of the 10 runs and from its distribution the error on the error can be roughly determined. For RNI we get for a fixed $m$ always the same topological susceptibility result. We estimate the error, as before, by the truncation error, here with a gauge value at $m_{0}=400$. The error of this error is negleced here. Results can be seen figure 4. For MCMC we observe the expected $1 / \sqrt{t}$ behavior, visualized by the red line in the figure. The RNI error scaling appears to not be exponential because we are considering values of $m$ that are too small for the asymptotic error scaling to be observed. However, although RNI is not yet in that regime, it already outperforms MCMC by orders of magnitudes.

\section{Conclusion}

In this paper we have applied the method of Recursive Numerical Integration with Gaussquadrature to a quantum mechnical system, namely the topological rotor. RNI is a method to numerically compute a high dimensional integral. It uses the structure of the integrand, here the next-neighbor coupling, to convert the high dimensional integral in many recursively computed small dimensional integrals which can be solved with high precision by using Gauss quadrature. 
The topological rotor model is simple enough to perform a first test of the RNI method but it also shows already some characteristic features of spin systems and even gauge theories. We compute the width of the distribution of the topological charge, the topological susceptibility as a benchmark quantity. There we find an exponentially fast error scaling. Although theoretically the error scaling should be even faster than exponential, we attribute this finding to the fact that we still work in an intermediate range of integration points and are not yet in the asymptotic regime. Comparing RNI directly with the for this model optimal MCMC Cluster algorithm shows an improvement of the

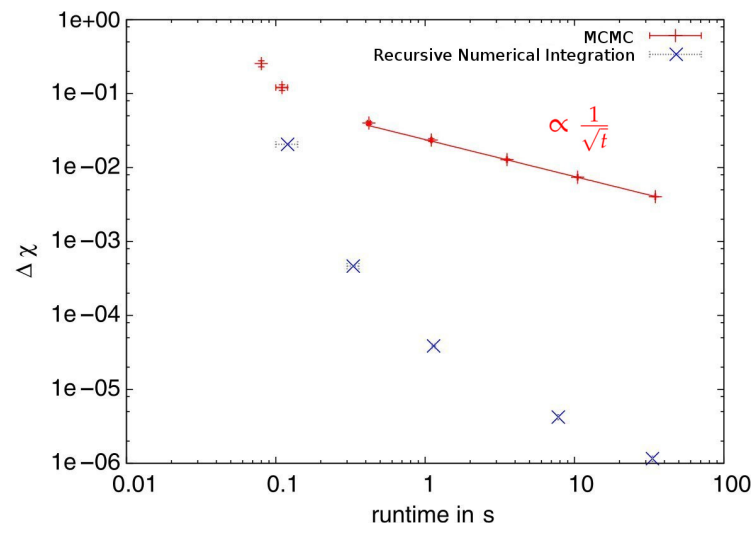

Figure 4: We show the run-time $t$ in seconds needed for the Cluster MCMC algorithm and RNI with Gauss quadrature to get a given error on the topological susceptibility on a stand-alone computer. The red line shows the expected $1 / \sqrt{t}$ behavior of MCMC.

error for RNI of several orders of magnitude even for a number of integration points where we are not yet in the intermediate, exponentially fast error scaling regime. Further applications of RNI to the anharmonic oscillator are reported in [9] and show extremely good results over a very broad range of parameters.

In symmary, RNI turns out to be an alternative method to MCMC because it leads to greatly improved error scaling and to order of magnitude reduced errors for a given runtime. This is crucial, especially in simulations with a larger number of dimensions. On the other side, applying RNI to a system with a larger number of dimensions results in the problem that the number of next neighbors doubles and instead of matrices $M^{i j}$ we have to deal with tensors $M^{i j k l}$ which makes the whole computation not feasible any more. Therefore we hope to combine this method with other techniques which are more suitable for higher dimensions to exploit the Gauss-quadrature error scaling to speed up computions.

\section{References}

[1] M. Lüscher, Computational Strategies in Lattice QCD, 2010, [1 002 . 4232].

[2] A. Genz and D. K. Kahaner, J. Comput. Appl. Math. 16 (1986) 255-258.

[3] A. Hayter, J. Statist. Plann. Inference 136 (2006) 2284-2296.

[4] H. Rothe, World Sci.Lect.Notes Phys. 43 (1992) 1-381.

[5] I. Montvay and G. Münster. Cambridge Monographs on Mathematical Physics. Cambridge University Press, 1994.

[6] C. Gattringer and C. B. Lang, Lect.Notes Phys. 788 (2010) 1-211.

[7] W. Bietenholz, U. Gerber, M. Pepe and U.-J. Wiese, JHEP 1012 (2010) 020, [1 009.2146$].$

[8] F. Niedermayer, Lect.Notes Phys. 501 (1998) 36, [hep-lat/9704009].

[9] A. Ammon, A. Genz, T. Hartung, K. Jansen, H. Leövey and J. Volmer, Comput. Phys. Comm. 198 (2016) $71-81$. 